\section{DIMENSIONANDO LA PRECARIEDAD URBANA Y SU VÍNCULO CON LA GESTIÓN DEL RIESGO DE DESASTRES}

Juliette Marin $^{1,2, *}$, Ingrid Mendez ${ }^{3}$, Juan Pablo Sarmiento ${ }^{4}$

\section{RESUMEN}

Los procesos acelerados e informales de urbanización a nivel global y el aumento de personas viviendo en zonas expuestas a amenazas presentan un problema social mayor que necesita una relectura de la precariedad urbana desde una perspectiva de reducción de riesgo. El presente estudio aborda el análisis de 23 modelos de medición de precariedad urbanas, con el objetivo de caracterizar su multicausalidad, multidimensionalidad e identificar las tendencias de uso de las mismas. A través de la consideración de las diferentes dimensiones físicas, socioeconómicas, sociopolíticas y legales de la precariedad urbana, se busca relacionar estas dimensiones con factores de riesgo de desastre, para profundizar la comprensión de la interrelación de estos conceptos. Se muestra que, si bien estas dimensiones no están siempre relacionadas directamente a factores de riesgo, sus vínculos pueden potencializar la generación de riesgo que a su vez puede acentuar condiciones de precariedad, por lo que no pueden ser analizadas aisladamente. Se avanza en propuestas para la formulación de una herramienta de caracterización de precariedad urbana para la toma de decisiones y el desarrollo de propuestas de intervención territorial, que integre directamente el riesgo de desastres, representando más apropiadamente la multidimensionalidad de la precariedad.

\section{PALABRAS CLAVE}

Precariedad urbana, Riesgo de desastres, Asentamientos humanos, Asentamientos informales

\section{MEASURING URBAN PRECARIOUSNESS AND ITS RELATION TO DISASTER RISK MANAGEMENT}

\section{ABSTRACT}

Urban processes, characterized at the global level by their acceleration and informality, and the increase of people living in areas prone to hazards, present a major social issue that requires a re-reading of urban precariousness from a risk reduction perspective. By analyzing 23 urban settlement precariousness indicators, this article seeks to characterize the multicausality, multidimensionality and to identify trends in the use of precariousness models. Through the consideration of physical, socio-economic, socio-political and legal dimensions of urban precariousness, and the analysis of their connections with risk factors, we intend to deepen the understanding of the interrelation of these concepts. It is shown that, although these dimensions are not always directly related to risk factors, their links can potentiate the generation of risk that in turn can accentuate precarious conditions, so they should not be analyzed separately. Finally, the study contributes in the formulation of a tool for characterizing urban precariousness, for decision making and the development of territorial intervention plans, which directly integrate risk while representing more appropriately the multidimensionality of precariousness.

\section{KEYWORDS}

Urban precariousness, Risk, Human settlements, Informal settlements

\begin{abstract}
1. Programa de Riesgo Sísmico, Universidad de Chile, Santiago, Chile.
\end{abstract}

2. Programa de Reducción de Riesgos y Desastres CITRID, Universidad de Chile, Santiago, Chile.

3. Consultora independiente Quetzaltenango, Guatemala.

4. Extreme Events Institute, Florida International University, Miami FL, Estados Unidos.

*Autor de correspondencia: jmarin@dgf.uchile.cl

\section{RECIBIDO}

1 de junio de 2018

ACEPTADO

2 de julio de 2018

\section{PUBLICADO}

25 de julio de 2018

\section{Formato cita}

Recomendada (APA):

Marin, J., Mendez, I. y Sarmiento, J. P. (2018).

Dimensionando la precariedad urbana y su vínculo con la gestión del riesgo de desastres. Revista de Estudios

Latinoamericanos sobre Reducción del Riesgo de Desastres REDER, 2(2), pp.4-15

\section{() $(\Phi \otimes$}

Todos los artículos publicados en REDER siguen una política de Acceso

Abierto y se respaldan en una Licencia CreativeCommons Atribución-NoComercial 4.0 Internacional.

Revista de Estudios Latinoamericanos sobre Reducción del Riesgo de Desastres (REDER)

Diseño: Lupe Bezzina Tipografía: Hospital 


\section{INTRODUCCIÓN}

El proceso de urbanización a nivel global marca unas tendencias irreversibles. Desde 2008, más de la mitad de la población mundial es urbana, y se espera que esta cifra llegue a $60 \%$ para el año 2030. Este crecimiento ha tenido desarrollos dentro de los mecanismos formales de gobernabilidad existente así como fuera de ellos. Aquellos procesos fuera de la formalidad determinan un aumento acelerado de los asentamientos precarios y son una parte considerable de los procesos de urbanización: se estima que $70 \%$ de las ciudades latinoamericanas provienen de tomas de terrenos ilegales, no planificadas e informales (Mertins, 2009). En países del "Sur Global", los asentamientos precarios reflejan una compleja problemática y un déficit de desarrollo aun no suficientemente dimensionado, cuestionando los límites de las políticas de planificación territorial. En países del "Norte Global", el tema vuelve a tener vigencia, bajo contextos de crisis migratorias y precarización asociados a políticas neoliberales implementadas en las décadas previas.

Los problemas de seguridad, de medios de vida y de acceso a educación y salud, así como la concentración de oportunidades en las ciudades ha resultado en la necesidad de migrar a centros urbanos de mayor tamaño, ubicándose frecuentemente en áreas no aptas para vivir, con deficiencias en acceso y calidad de servicios básicos, en zonas de alta fragilidad ambiental o expuestas a amenazas socionaturales, generando con ello asentamientos en situación de precariedad urbana, y nuevas zonas de riesgo a desastres. Estos procesos dinámicos, de retroalimentación, exigen nuevos análisis de las relaciones conceptuales de los fenómenos en juego.

Si bien es innegable la relación entre asentamientos precarios y riesgo de desastres, hay necesidad de profundizar en la multicausalidad, y para ello se requiere de una aproximación interdisciplinaria e intersectorial. El objetivo del presente artículo es contribuir en esta perspectiva, a través del estudio de las relaciones entre asentamientos precarios y los factores del riesgo de desastres, y de la propuesta de un modelo conceptual que permite caracterizar estas relaciones.

\section{ASENTAMIENTOS PRECARIOS Y PROCESOS DE CONSTRUCCIÓN DEL RIESGO}

La relación entre ciudad y procesos de generación de riesgo ha sido estudiada por diversos autores en particular desde los años 1990, ver por ejemplo Mansilla (2000).

Sin embargo, la consideración de asentamientos precarios como un objeto específico desde una perspectiva de riesgo en América Latina es más reciente. Como la señala Castro et al. (2015), existen estudios de las relaciones entre crecimiento económico, pobreza, informalidad, vulnerabilidad urbana, pero pocos centrados en la región latinoamericana, una de las más urbanizada del mundo, quizás por considerar acabados los procesos de formación de asentamientos informales debidos principalmente a la migración rural-urbana. En un estudio integral del riesgo en asentamientos informales en dos ciudades intermedias chilenas, los autores ponen en evidencia la necesidad de profundizar en trabajos sobre las características específicas de la vulnerabilidad relacionadas a asentamientos informales, proponiendo avanzar en estrategias de gestión del riesgo urbano que incluyan enfoques prospectivos (evitar construir riesgo), correctivos (gestionar el riesgo construido) y compensatorios (transferencia de riesgo y fortalecimiento de la resiliencia).

Sandoval y Sarmiento (2018) proponen una visión de la consideración de los asentamientos informales en América Latina y el Caribe con respecto a la gobernanza y resiliencia urbana para la reducción del riesgo de desastre, a partir de los informes nacionales elaborados para el trabajo de Habitat III. Los autores concluyen que, si bien existe un reconocimiento de la vinculación entre riesgo de desastre y asentamientos informales, así como de la importancia de la gobernanza y el fortalecimiento de la resiliencia, esto no se refleja en medidas concretas de acción y las referencias al Marco de Sendai son escasas.

\section{METODOLOGÍA DEL ESTUDIO}

Para la elaboración de un marco conceptual de precariedad urbana de asentamientos, con una perspectiva de riesgo de desastres, se analizaron 23 instrumentos de caracterización de precariedad habitacional, precariedad urbana, precariedad de la vivienda, o habitabilidad. La selección de las herramientas revisadas no es exhaustiva, y no pretende una caracterización cuantitativa de los instrumentos existentes. Lo que se busca es una identificación de cómo estos instrumentos de caracterización de asentamientos precarios han abordado la problemática del riesgo de desastres. 
La muestra considerada fue seleccionada con el fin de cumplir los siguientes criterios:

- $\quad$ Diversidad de autores del estudio y usuarios del instrumento: instituciones públicas técnicas, organizaciones no-gubernamentales, investigadores y académicos, organismos internacionales;

- $\quad$ Diversidad de propósitos del instrumento: diseño de planes, priorización de planes, inversión, investigación académica;

- Diversidad de localización del estudio: se consideraron casos de diferentes países de América, Europa y Oceanía, con una predominancia de estudios relativos a América Latina;

- Diversidad de escala del estudio: escala de la vivienda u hogar, conjuntos de viviendas, barrio o asentamiento, ciudad, región, país, continente, escala global.

Las condiciones de precariedad urbana están estrechamente vinculadas al contexto en el cual se generan, por lo que es de esperar que varíen los esfuerzos metodológicos dirigidos a caracterizar y/o establecer un nivel determinado de precariedad.

Por otro lado, a pesar de las diferencias en los procesos históricos, modelos económicos, formas de urbanización y condiciones de riesgo de los países de Latinoamérica, es posible establecer una perspectiva regional sobre precariedad. Hay en la región un registro de décadas de estudios y planes sobre esta temática, hecho que facilita la generación de una comprensión del fenómeno de precariedad de la vivienda y del asentamiento como colectivo, así como aprendizajes acerca de las diferentes estrategias que han sido llevadas a cabo. Es por ello que se decidió dar un énfasis a casos latinoamericanos.

A partir del conjunto estudiado, se trabajó primero en analizar el tipo de metodología utilizada en la construcción de los instrumentos, los componentes de definición de éstos, el tipo y origen de datos usados. Se buscó luego identificar tendencias, particularidades y divergencias en la conceptualización de la precariedad de asentamientos, a partir de un análisis de las variables y componentes de definición de ésta, evidenciando la multicausalidad y complejidad del concepto estudiado. Finalmente se analizó la relación de estas variables y componentes con factores del riesgo de desastres.

REVISIÓN DE MODELOS SOBRE PRECARIEDAD HABITACIONAL

La Tabla 1 presenta los indicadores de precariedad considerados en el estudio.

\begin{tabular}{|c|c|c|c|c|}
\hline & Nombre instrumento & Contexto de uso & País o Región & Referencia \\
\hline 1 & $\begin{array}{l}\text { Indicadores de Pobreza y } \\
\text { Precariedad }\end{array}$ & Estudio de la CEPAL & América Latina & $\begin{array}{l}\text { Pobreza y precariedad del hábitat en ciudades de América } \\
\text { Latina y el Caribe. Mac Donald, J. (2004). CEPAL. }\end{array}$ \\
\hline 2 & Cartografía de los tugurios & Estudio de la CEPAL & América Latina & $\begin{array}{l}\text { Metas del Milenio y tugurios: una metodología utilizando } \\
\text { datos censales. Candia, D. (2005). CEPAL. }\end{array}$ \\
\hline 3 & $\begin{array}{l}\text { Catastro de asentamientos } \\
\text { precarios }\end{array}$ & Investigación académica & América Latina & $\begin{array}{l}\text { Catastros de asentamientos precarios en América Latina: } \\
\text { consideraciones metodológicas e implicancias de política. } \\
\text { Atria, J., Pérez I. (2015) Revista de temas contemporá- } \\
\text { neos sobre lugares, política y cultura, 5, (julio-diciembre). }\end{array}$ \\
\hline \multirow[t]{2}{*}{4} & Indicador de precariedad & $\begin{array}{l}\text { Programa habitacional de } \\
\text { Carlota }\end{array}$ & Argentina & $\begin{array}{l}\text { Medición de la precariedad en asentamientos urbanos } \\
\text { irregulares. Estudio comparativo entre Venezuela, Chile y } \\
\text { Argentina. Tapia, R. (2000). Revista INVI, 15(40). }\end{array}$ \\
\hline & & & & $\begin{array}{l}\text { Hacia la construcción de indicadores de precariedad con } \\
\text { una visión sistémica. Enet, M. (1999). CEVE. }\end{array}$ \\
\hline 5 & $\begin{array}{l}\text { Condiciones habitacionales } \\
\text { y Calidad de vida urbana }\end{array}$ & Investigación académica & Argentina & $\begin{array}{l}\text { Condiciones habitacionales y Calidad de vida urbana. El } \\
\text { caso de la ciudad de Bahía Blanca. Prieto, M.B. (2007). En: } \\
\text { IX Jornadas Argentinas de Estudios de Población (AEPA). }\end{array}$ \\
\hline 6 & $\begin{array}{l}\text { Medición de asequibilidad } \\
\text { de la vivienda }\end{array}$ & $\begin{array}{l}\text { ONG Fundação João } \\
\text { Pinheiro }\end{array}$ & Brasil & $\begin{array}{l}\text { Measuring Housing Affordability in Sao Paulo } \\
\text { Metropolitan Region. Acolin, A. y R. Green. (2015). } \\
\text { Disponible en SSRN: https://ssrn.com/abstract=2553488 } \\
\text { o http://dx.doi.org/10.2139/ssrn.2553488 }\end{array}$ \\
\hline 7 & $\begin{array}{l}\text { Indicadores relacionados a } \\
\text { la vivienda }\end{array}$ & $\begin{array}{l}\text { Ministerio de Desarrollo } \\
\text { Social }\end{array}$ & Chile & $\begin{array}{l}\text { Página Web. Consultada el 26/03/2017. http://obser- } \\
\text { vatorio.ministeriodesarrollosocial.gob.cl/casen/case } \\
\text { n_def_vivienda.php }\end{array}$ \\
\hline 8 & $\begin{array}{l}\text { Catastro de los campa- } \\
\text { mentos y asentamientos } \\
\text { irregulares }\end{array}$ & $\begin{array}{l}\text { Catastro Nacional de } \\
\text { Asentamientos Precarios }\end{array}$ & Chile & $\begin{array}{l}\text { Medición de la precariedad en asentamientos urbanos } \\
\text { irregulares. Estudio comparativo entre Venezuela, Chile y } \\
\text { Argentina. Tapia, R. (2000). Revista INVI, 15(40). }\end{array}$ \\
\hline
\end{tabular}

(Continúa en la siguiente página) 


\begin{tabular}{lllll} 
& Nombre instrumento & Contexto de uso & País o Región & Referencia \\
\hline 9 & Bienestar social & Investigación académica & Chile & $\begin{array}{l}\text { Bienestar habitacional. Guía de diseño para un hábitat } \\
\text { residencial sustentable. Jirón, P., Toro, A., Caquimbo, S., } \\
\text { Goldsack, L., Martinez, L., Colonelli, P., Hornazábal, N. y P. } \\
\text { Sarmiento. (2004). ISBN: 956-19-0444-6 }\end{array}$ \\
\hline 10 & $\begin{array}{l}\text { Indices para caracterizar los } \\
\text { campamentos }\end{array}$ & ONG Techo & Chile & $\begin{array}{l}\text { Datos duros de una realidad muchísimo más dura. } \\
\text { Informe Encuesta Nacional de Campamentos 2015, } \\
\text { Centro de Investigación Social de TECHO-CHILE. (2015). }\end{array}$ \\
\hline 11 Índice de Precariedad \\
$\begin{array}{l}\text { Urbana en Asentamientos } \\
\text { Precarios }\end{array}$ & $\begin{array}{l}\text { Observatorio Metropolitano } \\
\text { del Área Metropolitana de } \\
\text { Bucaramanga }\end{array}$ & Colombia & $\begin{array}{l}\text { Página Web. Consultada el 26/03/2017. http://www. } \\
\text { observatoriometropolitano.com.co/ }\end{array}$
\end{tabular}

\begin{tabular}{ll}
\hline 12 & $\begin{array}{l}\text { Indicadores para análisis } \\
\text { situacional de asentamientos } \\
\text { precarios }\end{array}$
\end{tabular}

Covarino S., Mansilla M., Zurita C. (2010). Universidad Rafael Landivar. Guía de Diagnóstico Urbano. Participativo para asentamientos precarios con enfoque en la Meta 11 de los ODM. Guatemala

\begin{tabular}{lll}
\hline Indicador para la carac- & Programa Barrio mío de & Guatemala \\
terización por comunidad & Mixco \\
en relación a criterios por &
\end{tabular}

Presentación "Transformando comunidades de alto riesgo a comunidades seguras, productivas y resilientes con la participación activa de familias y autoridades en vulnerabilidad la búsqueda de un desarrollo sostenible", USAID - PCI Guatemala, 2017

\begin{tabular}{|c|c|c|c|c|}
\hline 14 & Mapa del Déficit Habitacional & $\begin{array}{l}\text { Instituto Nacional de } \\
\text { Estadísticas e Informática }\end{array}$ & Perú & $\begin{array}{l}\text { Mapa del Déficit Habitacional a Nivel Distrital, } 2007 . \\
\text { Instituto Nacional de Estadísticas e Informática (2007). }\end{array}$ \\
\hline 15 & $\begin{array}{l}\text { Indicador de precariedad a } \\
\text { nivel de unidades de plani- } \\
\text { ficación física y unidades de } \\
\text { diseño urbano }\end{array}$ & $\begin{array}{l}\text { Programa Promueba } \\
\text { Caracas }\end{array}$ & Venezuela & $\begin{array}{l}\text { Medición de la precariedad en asentamientos urbanos } \\
\text { irregulares. Estudio comparativo entre Venezuela, Chile y } \\
\text { Argentina. Tapia, R. (2000). Revista INVI, 15(40). }\end{array}$ \\
\hline 16 & $\begin{array}{l}\text { Características de asenta- } \\
\text { mientos precarios }\end{array}$ & UN Habitat & Global & UN Habitat (2015) \\
\hline 17 & $\begin{array}{l}\text { Vínculos entre viviendas } \\
\text { precarias y desigualdades } \\
\text { en salud }\end{array}$ & Investigación académica & Australia & $\begin{array}{l}\text { Precarious housing and health inequalities: What are the } \\
\text { links? S Mallett, R Bentley, E Baker, K Mason, D Keys, V } \\
\text { Kolar \& L Krnjacki, Hanover Welfare Services, University } \\
\text { of Melbourne, University of Adelaide, Melbourne } \\
\text { Citymission, Australia. (2011) }\end{array}$ \\
\hline 18 & $\begin{array}{l}\text { Análisis etnográfico institu- } \\
\text { cional de las relaciones entre } \\
\text { seguridad de los barrios, } \\
\text { precariedad de la vivienda y } \\
\text { procesos de exclusión }\end{array}$ & Investigación académica & Canadá & $\begin{array}{l}\text { Community Safety, Housing Precariousness and } \\
\text { Processes of Exclusion: An Institutional Ethnography } \\
\text { from the Standpoints of Youth in an 'Unsafe' Urban } \\
\text { Neighbourhood. Nichols, N. \& J. Braimoh. (2016) Critical } \\
\text { Sociology 1-16. DOI: 10.1177/0896920516658941 }\end{array}$ \\
\hline 19 & $\begin{array}{l}\text { Indicador de precariedad de } \\
\text { vivienda }\end{array}$ & $\begin{array}{l}\text { Estudio del Wellesley } \\
\text { Institute }\end{array}$ & Canadá & $\begin{array}{l}\text { Precarious Housing in Canada 2010. Wellesley Institute. } \\
\text { (2010) }\end{array}$ \\
\hline 20 & $\begin{array}{l}\text { Indicador de Condición de } \\
\text { Viviendas }\end{array}$ & Homeland security & EEUU & $\begin{array}{l}\text { Draft Interagency Concept for Community Resilience } \\
\text { Indicators and National-Level Measures. Mitigation } \\
\text { Framework Leadership Group (MitFLG) Draft Concept } \\
\text { Paper. Homeland security. (2016) }\end{array}$ \\
\hline 21 & $\begin{array}{l}\text { Indicador de Asequibilidad a } \\
\text { la Vivienda }\end{array}$ & Homeland security & EEUU & $\begin{array}{l}\text { Draft Interagency Concept for Community Resilience } \\
\text { Indicators and National-Level Measures. Mitigation } \\
\text { Framework Leadership Group (MitFLG) Draft Concept } \\
\text { Paper. Homeland security. (2016) }\end{array}$ \\
\hline \multirow[t]{3}{*}{22} & $\begin{array}{l}\text { Indicador de precariedad de } \\
\text { la vivienda }\end{array}$ & Investigación académica & Europa & $\begin{array}{l}\text { Constructing a Housing Precariousness Measure for } \\
\text { Europe. Clair, A., Reeves, A., McKee, M. and D. Stuckler. } \\
\text { (2017). }\end{array}$ \\
\hline & & & & $\begin{array}{l}\text { Housing precariousness: its measurement and impact } \\
\text { on health before and after the Great Recession. Clair, A., } \\
\text { Reeves, A., McKee, M. and D. Stuckler. (2016). European } \\
\text { Journal of Public Health, Vol. 26, Supplement } 1 \text {. }\end{array}$ \\
\hline & & & & $\begin{array}{l}\text { Living on the edge: precariousness and why it matters } \\
\text { for health. McKee, M., Reeves, A., Clair A. And D. Stuckler } \\
\text { (2017). Archives of Public Health (2017) 75:13. DOI } \\
\text { 10.1186/s13690- 017-0183-y }\end{array}$ \\
\hline 23 & $\begin{array}{l}\text { Características del mal } \\
\text { alojamiento }\end{array}$ & ONG Abbé Pierre & Francia, UE. & $\begin{array}{l}\text { Fondation Abbé-Pierre. L'état du mal-logement en } \\
\text { France 2017. Rapport annuel \#23. (2018) }\end{array}$ \\
\hline
\end{tabular}

Tabla 1. Indicadores del estudio

Fuente: Autores, 2018 
Primero, en todos los estudios se define la precariedad a través de un conjunto de factores de muy variadas características; la precariedad no aparece como un concepto que se pueda definir directamente y, por ende, medir por si mismo, sino que requiere la definición y evaluación de otros componentes, que a su vez pueden ser definidos por subcomponentes.

De la revisión de los modelos resalta la importancia de las metodologías cuantitativas, observándose una diferencia entre la aproximación realizada en Latinoamérica y la diseñada en países desarrollados. En el primer caso (instrumentos 1 a 15 de la Tabla 1), se tiende a buscar un indicador numérico final o único que refleje el estado de precariedad de una vivienda, barrio o proyecto urbano. En el segundo caso (instrumentos 17 a 23 de la Tabla 1), se buscan medir las diferentes dimensiones que convergen en el concepto de precariedad.

Esta diferencia de abordaje de la precariedad se entiende por el uso que se les da a las herramientas consideradas: en el primer caso, de países de Latinoamérica, se trata principalmente de evaluar el estado e impacto de proyectos de intervención en vista de disminuir la precariedad habitacional; en el segundo caso, de países del "Norte Global", se trata más bien de aproximarse a una interseccionalidad donde el problema habitacional se relaciona con otros problemas sociales como por ejemplo de salud pública, discriminación social, gentrificación.

En la mayoría de los instrumentos estudiados, los datos de entrada corresponden principalmente a información secundaria - datos censales, datos de organismos gubernamentales relacionados a problemáticas de urbanismo o vivienda, catastros municipales, regionales, nacionales o transnacionales, fotografías aéreas - aunque también se reconoce el uso de datos primarios como entrevistas o encuestas para construir algunos subcomponentes de la precariedad habitacional.

Entre los diferentes componentes usados para definir la precariedad prevalecen los componentes constructivos y/o físicos -tipo de estructuras, materialidad, conexiones a redes y servicios, presencia de infraestructura adecuada, etc.-, componentes socioeconómicos -acceso al empleo, a servicios de educación y salud, etc.-, y componentes sociopolíticos y legales -existencia de normas, planes sociales, tipos de propiedad, entre otros.

Para un estudio más preciso de la definición de los componentes de la precariedad habitacional, se propone considerar ocho dimensiones, que permitan una mejor descripción de lo que cada una busca caracterizar. Estas dimensiones se establecieron para representar exhaustivamente todos los factores considerados en la caracterización de la precariedad urbana del conjunto de referencia. Las dimensiones son:

(a) las condiciones físicas de la vivienda;

(b) las condiciones de aceso de servicios básicos domiciliarios;

(c) condiciones de acceso a otros servicios;

(d) características de hacinamiento;

(e) condiciones de asequibilidad económica;

(f) condiciones de acceso a otros servicios;

(g) condiciones del entorno;

(h) características físico-sociales del entorno.

De este modo, se buscó comparar como cada instrumento considera las diferentes escalas que coexisten -hogar, vivienda, asentamiento por lo menos-, y las diferentes aristas del fenómeno estudiado -físicas, económicas, jurídicas, sociales y ambientales.

Las dimensiones estudiadas, y sus respectivas definiciones e indicadores aparecen en la Tabla 2 , en un orden de mayor a menor recurrencia en el conjunto de instrumentos considerado. Las tres primeras dimensiones en importancia en la caracterización de asentamientos precarios son dimensiones principalmente físicas, relacionadas a las condiciones físicas de la vivienda, el acceso a servicios básicos y al hacinamiento. Las siguientes dos dimensiones son mayormente de carácter legal y socioeconómico, donde la legalidad se refiere en América Latina en la posesión de un título de propiedad o la regularidad administrativa de la vivienda. Las tres últimas dimensiones refieren a características desde una escala de asentamiento o barrio, tales como el acceso a diversos 
servicios urbanos (educación, salud, cultura, etc.), condiciones del entorno y características físicas, sociales e históricas del asentamiento.

\begin{tabular}{|c|c|c|}
\hline & Dimensión & Descripción de la dimensión \\
\hline a & $\begin{array}{l}\text { Condiciones físicas de la } \\
\text { vivienda }\end{array}$ & $\begin{array}{l}\text { Condiciones relacionadas a características físicas de la construcción de } \\
\text { la vivienda, tales como tipo de estructura, materiales de construcción, } \\
\text { superficie, plomería, baños, o la calidad de la construcción en términos } \\
\text { térmicos, de humedad, }\end{array}$ \\
\hline$b$ & $\begin{array}{l}\text { Condiciones de acceso } \\
\text { a servicios básicos } \\
\text { domiciliarios }\end{array}$ & $\begin{array}{l}\text { Condiciones relacionadas a la conexión y el acceso de servicios básicos } \\
\text { domiciliarios, es decir aquellos que sirven para satisfacer las necesidades } \\
\text { básicas de bienestar y salubridad de la población, como lo son agua } \\
\text { potable, saneamiento y electricidad. }\end{array}$ \\
\hline c & Condición de hacinamiento & $\begin{array}{l}\text { Relación entre el número de personas en una vivienda y el espacio o } \\
\text { número de cuartos disponibles. }\end{array}$ \\
\hline$d$ & $\begin{array}{l}\text { Condiciones de tenencia y } \\
\text { seguridad }\end{array}$ & $\begin{array}{l}\text { Condiciones jurídicas, administrativas, normativas y legales que aseguran } \\
\text { la mantención de la vivienda. Se considera por ejemplo la existencia de } \\
\text { un título de propiedad de la vivienda, información catastral, estatus de } \\
\text { ocupación o estatus tributario. }\end{array}$ \\
\hline e & $\begin{array}{l}\text { Condiciones de } \\
\text { asequibilidad económica }\end{array}$ & $\begin{array}{l}\text { Condiciones socioeconómicas relacionadas a las capacidades de obtención } \\
\text { y mantención de la vivienda. Se considera por ejemplo los ingresos del } \\
\text { hogar, su capacidad de cubrir los costos de vivienda, la disponibilidad de } \\
\text { eventuales apoyos para la mantención. }\end{array}$ \\
\hline$f$ & $\begin{array}{l}\text { Condiciones de acceso a } \\
\text { otros servicios }\end{array}$ & $\begin{array}{l}\text { Condiciones relacionadas al acceso a servicios otros que los servicios } \\
\text { básicos domiciliarios, que atiende necesidades de las personas y/o } \\
\text { sociedad en conjunto. Se considera por ejemplo los servicios de } \\
\text { transporte, educación, salud, justicia, espacios culturales, espacios } \\
\text { recreativos. Estos servicios pueden ser públicos o privados. }\end{array}$ \\
\hline g & Condiciones del entorno & $\begin{array}{l}\text { Condiciones propias del entorno que rodea los habitantes o en que se } \\
\text { desarrollan actividades y que puede tener un impacto en su forma } \\
\text { de habitar. Se considera por ejemplo el tipo de suelo, la exposición a } \\
\text { amenazas, la ubicación con respecto a industrias contaminantes, la } \\
\text { topografía. }\end{array}$ \\
\hline h & $\begin{array}{l}\text { Características físicas y } \\
\text { sociales del asentamiento }\end{array}$ & $\begin{array}{l}\text { Variables descriptivas del tipo de asentamiento y de la población de éste, } \\
\text { que van más allá de la escala de la vivienda. Se consideran variables } \\
\text { físicas tales como la superficie del asentamiento o la presencia de } \\
\text { espacios verdes; y variables sociales como la edad del asentamiento, la } \\
\text { existencia de organizaciones, los niveles de ingresos. }\end{array}$ \\
\hline
\end{tabular}

Tabla 2. Dimensiones de los indicadores de precariedad de asentamientos Fuente: Autores, 2018

En este proceso de descomposición de los diferentes indicadores de precariedad de asentamientos, se evidencia que las dimensiones identificadas se encuentran interconectadas y que no deben ser analizadas aisladamente, sino integradamente. La situación de precariedad urbana como ya se ha dicho es el resultado de un proceso multicausal y por tanto requiere de un análisis profundo en los aspectos sociales, ambientales, económicos y políticos.

La Figura 1 ilustra de manera gráfica los ejes esenciales identificados, partiendo desde las dimensiones más comunes hasta las menos utilizadas. La frecuencia de uso no necesariamente refleja la importancia de las variables en la medición de la precariedad.

Durante la revisión bibliográfica se hizo evidente la conexión de la problemática de precariedad urbana con un amplio panorama de problemáticas sociales tales como empleo y precariedad laboral; ingresos por hogar y desigualdad; violencia; acceso a la justicia; criminalización de la violencia juvenil; salud pública; gentrificación; raza y clase. Esta multidimensionalidad se relaciona con una multicausalidad de la precariedad urbana, la cual se refleja en la mayoría de los modelos estudiados. De este modo, es relevante preguntarse en qué medida la multidimensionalidad de la precariedad urbana puede ser adecuadamente representada a través de un indicador único.

La Figura 2 representa factores relacionados a la precariedad de asentamientos según los estudios consultados, distinguiendo según el tipo de vínculo: 1) el factor agravante, el cual genera, contribuye o acentúa la precariedad habitacional, y 2) el factor de impacto, generado o influenciado por la precariedad habitacional. Esto demuestra la multidimensionalidad de la precariedad urbana que se relaciona con diversos elementos estructurantes de las políticas de desarrollo. 


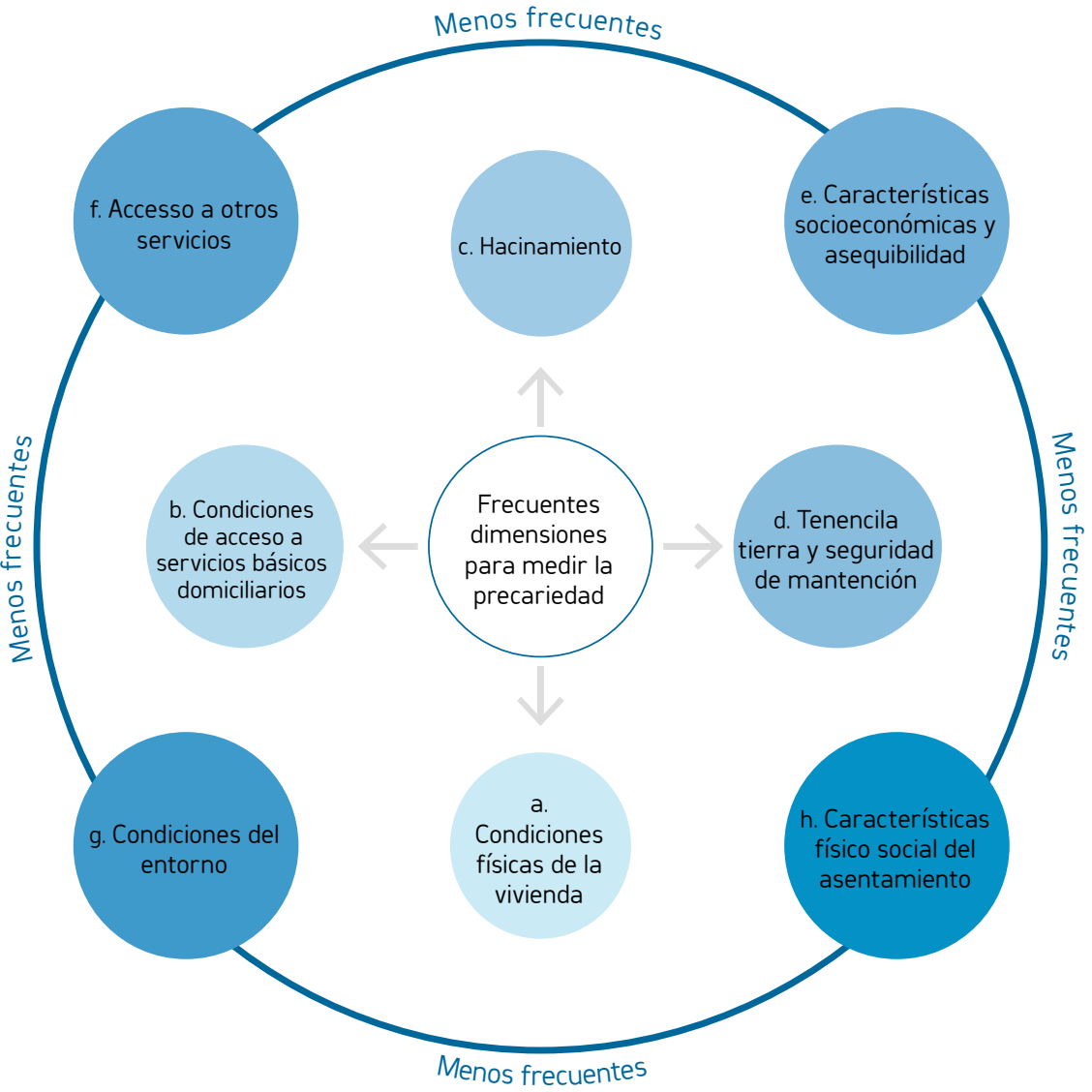

Figura 1. Dimensiones frecuentes y las menos frecuentes utilizados en los instrumentos analizados Fuente: Autores, 2018 Autoconstrucción Precio materiales construcción Deficiencia de planes/normas públicas Fallas de mercados (agua, cloacas, etc.)

$$
\text { Pobreza Expulsiones }
$$$$
\text { Migraciones }
$$
Crecimiento poblacional

Existencia y conocimiento de Informalidad dispositivos legales de tenencia Migraciones

Acceso a la administración y justicia Legislación suelo Crisis económica Migraciones y vivienda Pobreza y desigualdad

Flexibilización laboral y pauperización$$
\text { Deficiencia de planes/normas públicas }
$$

Fallas de mercados

Alejamiento geográfico

Expansión urbana

Regulación uso y precio de suelos

llegalidad tenencia y construcción

Historia y edad del asentamiento

Superficie, localización del asentamiento
Dimensiones de la precariedad

a. Condiciones físicas de la vivienda

b. Condiciones de acceso a servicios básicos domiciliarios

\section{c. Condiciones de} hacinamiento

d. Condiciones de tenencia seguridad

\section{e. Condiciones de} asequibilidad económica

f. Falta de acceso a servicios urbanos

Condiciones del entorno

h. Características físicas y sociales del asentamiento
Ruido, confort térmico, comodidad

Impacto en la salud (física, mental)

Capacidad de concentración, sueño, estudio

Impacto en el entorno (contaminación de suelos, basurales, etc.) Impacto en la salud (física, mental)

Costos excesivos, falta de asequibilidad

Falta de intimidad/privacidad Impacto en la salud (física, mental)

Capacidad de concentración, sueño, estudio

Percepción de inseguridad

$$
\text { llegalidad Marginalidad }
$$

Acceso a la justicia

Infrasubsistencia Inseguridad

Racismo Falta movilidad geográfica

Discriminación Acceso a los mercados

Discriminación

Deficiencia de movilidad urbana Impacto en la salud, falta de acceso al

Segregación Precariedad laboral

Zonas expuestas a amenazas

mpacto ambiental

Percepción del asentamiento (basurales, contaminación)

Carencia de espacios verdes/públicos

Percepción del asentamiento

Sentimiento de identidad y comunidad

Resiliencia comunitaria 
La Figura 2 permite mostrar la relación entre una dimensión recurrente de caracterización de precariedad urbana con factores de muy diferentes características que estas mismas dimensiones.

Consideremos, por ejemplo, la dimensión b. de Condiciones de acceso a servicios básicos domiciliarios, principalmente agua potable, saneamiento y electricidad. Entre los factores agravantes de malas condiciones de acceso a servicios básicos domiciliarios, se identifican la deficiencia, por ausencia o ineficiencia, de planes y normativas públicas que permitan asegurar la existencia de infraestructura y distribuidores de estos servicios básicos; o la deficiencia de planificación urbana que lleve a generación de nuevos asentamientos en zonas provistas de dichos servicios. Las fallas en el mercado en cuanto a la distribución equitativa y accesible a los servicios domiciliarios también son un factor agravante, particularmente en países con mercados de servicios básicos privatizados, como en Chile.

Las condiciones deficientes de acceso a servicios domiciliarios básicos impactan directamente en la calidad de vida de los habitantes de estos asentamientos, con un impacto en la salud física y mental. La búsqueda de soluciones alternativas a las formales no asequibles, propicia un uso no apto del entorno, por ejemplo, con la evacuación de cloacas en puntos de aguas o uso de quebradas para basurales. Esto impacta negativamente el ambiente y paisaje del área, lo cual a su vez conlleva a una nueva afectación en la salud física de la población y en la percepción de su entorno. Al no tener acceso servicios regulados, los habitantes de asentamientos precarios también están expuestos por estos servicios a tarifas fuera de la normalidad, que pueden ser excesivas, afectando la economía del hogar.

De este modo, la dimensión b. se mide generalmente con la presencia o no de conexiones a servicios básicos, pero engloba de manera no lineal causas y consecuencias físicas, legales, económicas, de diversas índoles que van más allá de la conexión física con redes de infraestructuras.

Finalmente, se desarrolló un mapa conceptual de las dimensiones de la precariedad urbana considerando sus relaciones con el riesgo de desastres, ilustrado en la Figura 3. Esta figura refleja la complejidad de las vinculaciones entre diferentes procesos de precariedad urbana y riesgo de desastre, y lleva a interrogar sobre como los modelos de precariedad pueden integrar transversalmente una perspectiva de riesgo.

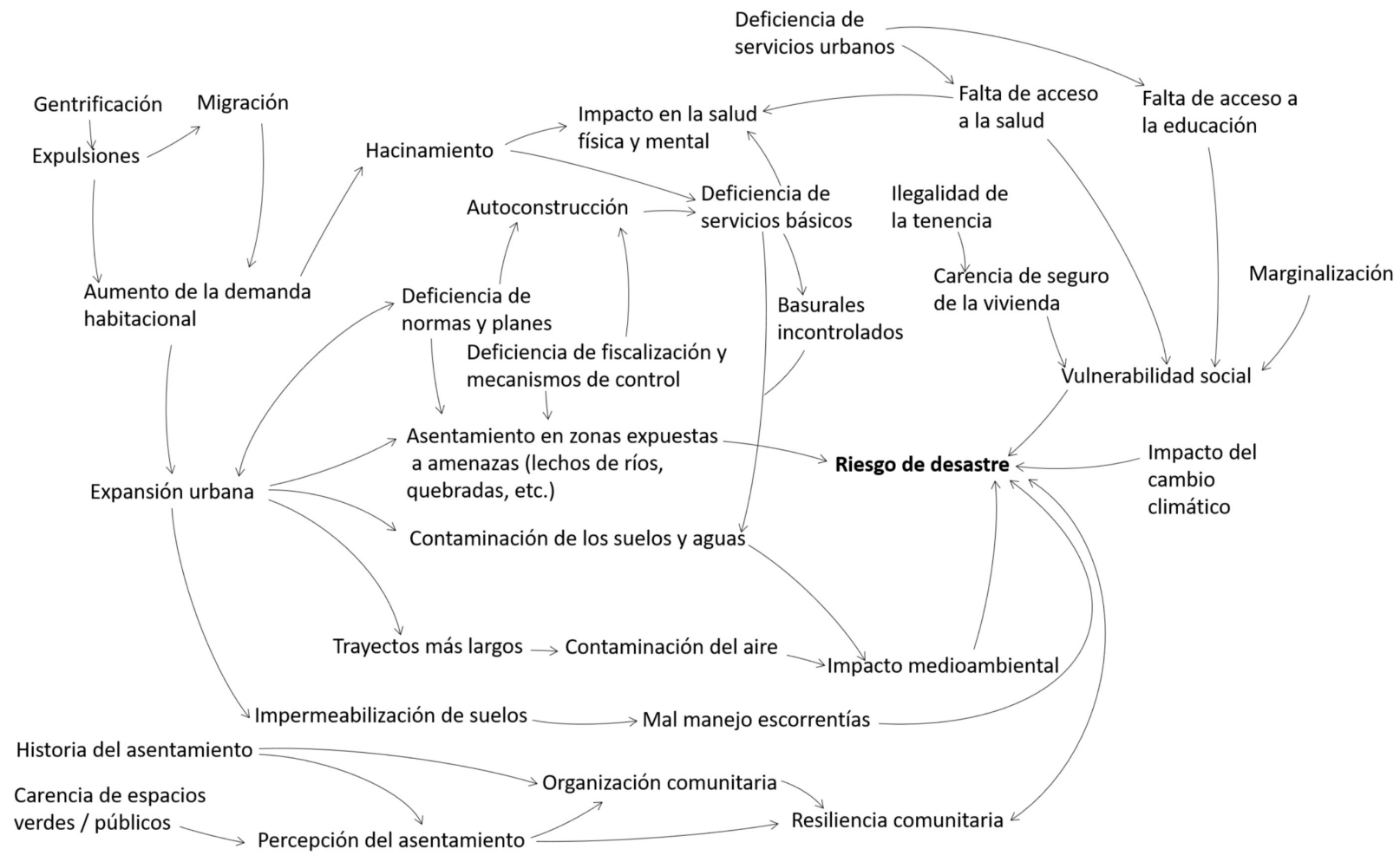


Se evidencia con este mapa conceptual que la vinculación existente entre todos los factores utilizados para la caracterización de la precariedad y el riesgo de desastre es compleja y lleva a abordar fenómenos considerados a menudo de forma fragmentada.

Por ejemplo, el hacinamiento se considera mayoritariamente en los estudios de precariedad de asentamientos, aunque su relación con el riesgo de desastre no se explicite. La Figura 3 permite evidenciar este vínculo. Efectivamente, el hacinamiento impacta las condiciones de salud de los habitantes, en tanto deben sobre-ocupar un espacio. También lleva a aumentar la demanda de servicios básicos, generalmente inexistentes formalmente, lo que genera una mayor presión de soluciones irregulares. Estas soluciones fuera de la normatividad y planificación de redes urbanas significan un uso impropio de los suelos, que puede traducirse en contaminación de suelos y aguas, así como en la generación de basurales incontrolados. Es decir en un impacto medioambiental negativo, que se relaciona directamente como un factor agravante del riesgo de desastre, generando mayor exposición de elementos sensibles para la población.

\section{MODELO CONCEPTUAL DE LA PRECARIEDAD HABITACIONAL PARA AMÉRICA LATINA}

El modelo conceptual a desarrollar busca caracterizar las relaciones entre factores asociados a la precariedad urbana y factores de riesgo de desastre. Se propone abordar la dificultad de formular un indicador único que represente fenómenos multidimensionales. Para ello, se propone definir criterios mensurables de precariedad, que corresponderían a los ejes o dimensiones fundamentales de la precariedad urbana. Una vez definidos éstos, se propone pensar en umbrales de alerta de precariedad: en cada una de estas dimensiones, si el indicador alcanza cierto umbral (criterio), se establece una alerta. La acumulación de alertas correspondería a un estado de precariedad. De este modo, un asentamiento que tenga una(s) de sus dimensiones como crítica(s) sería considerado un asentamiento precario, aunque la ponderación de dimensiones no lo muestre como tal. Este tipo de definición de precariedad no es usual en América Latina, del conjunto de instrumentos analizados sólo el instrumento 20 (Indicador de Condición de Viviendas, Homeland Security, Estados Unidos) propone tal enfoque multidimensional por umbral.

De los 23 instrumentos analizados, solo 2 indicadores incluyen explícitamente factores de riesgo de desastres en su caracterización de la precariedad, y un tercero lo incluye indirectamente: 1) El indicador para la caracterización por comunidad en relación a criterios por vulnerabilidad en el proyecto "Barrio Mío" en el Municipio de Mixco, en Guatemala, se incluyen variables como existencia de pendientes, laderas y zanjones, eventos naturales notables y contaminación del medio ambiente. 2) El Catastro Nacional de Asentamientos Precarios de Chile utiliza factores de riesgo ambiental (pendiente elevada, zona inundable) y factores de riesgo ambiental (basurales, fuentes de contaminación industrial, plantación forestal, riesgos acumulados). 3) El Plan de habilitación física para los barrios de Caracas (Inventario Nacional de Barrios de FUNDACOMUN-OCEI; Programa Promueba Caracas), por su parte, considera indirectamente la exposición a amenazas de origen natural al integrar las variables de altura del asentamiento con respecto al nivel del mar, tipos de suelos y clinometría (pendientes topográficas).

Considerando la vigencia de la articulación entre asentamientos precarios y construcción del riesgo, se propone incluir directamente el riesgo de desastres en la estimación de precariedad urbana. Si retomamos la propuesta de umbrales de precariedad, esto lleva directamente a formular la pregunta siguiente: ¿Cuál es el riesgo aceptable?

Por otro parte, la relación entre precariedad urbana y gobernanza está incluida en la mayoría de los modelos estudiados a través del tipo de propiedad de la vivienda, vinculado con la situación de legalidad y la seguridad de mantención en la vivienda (Indicadores 1, 2, 3, 4, 5, 6, 8, 9, 11, 12, 13, 15, 17, 19, 23 según la Tabla 1). Otros indicadores no incluyen la gobernanza (Indicadores 7, 10, 18, 20, 21, 22 según la Tabla 1). Es notable la diferencia de consideración de la situación de mantención en el hábitat, asociado a expulsiones o movimientos forzados en países del "Norte Global" y muy predominante asociado a legalidad en estudios de países Latinoamericanos, por las condiciones propias de sus procesos de urbanización. En los indicadores revisados, la gobernanza se vincula entonces con la institucionalidad, o la gobernabilidad. Sin embargo, estudios de resiliencia urbana ante desastres reconocen otras dimensiones de la gobernanza, como la cohesión social o la asociatividad (Sandoval \& Sarmiento, 2018), que interrogan la relación entre los diferentes actores sociales. Estas interpretaciones deberían incluirse en un modelo conceptual de precariedad urbana con consideración de riesgo de desastre. 


\section{DISCUSIÓN Y CONCLUSIÓN}

La gestión del riesgo está directamente relacionada con el desarrollo, planificación territorial, y los sistemas de gestión ambiental, que son fundamentales para el desarrollo social sostenible y para contrarrestar los índices de precariedad y los niveles de vulnerabilidad de la población. La precariedad y la vulnerabilidad urbana refuerzan la exposición de los asentamientos y sus habitantes ante fenómenos adversos y eventos extremos.

La precariedad de los asentamientos urbanos se constituye como un concepto compuesto, multidimensional, producto de una multicausalidad. Adicionalmente, gran parte de los asentamientos precarios se encuentran en condiciones de vulnerabilidad social, económica, política y ambiental, así como en condiciones medianas y altas de exposición a amenazas de origen natural o antrópico, propiciando condiciones de agudización del riesgo urbano. Esto constituye un desafío mayor tanto para las políticas urbanas como de los sistemas de reducción y gestión del riesgo a nivel de ciudades.

Si bien la construcción de indicadores de precariedad urbana en pocas ocasiones integra directamente el riesgo de desastres (particularmente la exposición a amenazas), si contempla diferentes factores de vulnerabilidad de estructuras y redes de servicios, y aborda la susceptibilidad económica, la marginalización, y la fragilidad de los mecanismos de gobernanza. Ante la urgencia de enfrentar el problema social mayor que plantea la reducción del riesgo en asentamientos precarios, nos parece esencial apuntar hacia la consideración de variables que midan integralmente el riesgo dentro de los indicadores de precariedad.

Por otro lado, a la luz de este estudio, dos aportes son propuestos en la conceptualización de la precariedad, en particular desde una perspectiva de reducción del riesgo. Primero, se sostiene que, dada la multidimensionalidad de los fenómenos estudiados, se debería apuntar a la construcción de indicadores por umbrales, integrando, como ya se mencionó, el riesgo de desastres. Por otro lado, aspectos de gobernanza fuera de la institucionalidad, tales como la cohesión social o las organizaciones comunitarias, deberían ser considerados.

Cada una de las dimensiones estudiadas puede contribuir a generar información para desarrollar análisis e instrumentos que propicien la toma de decisiones e implementación de acciones de atención de la precariedad bajo un enfoque integral de gestión de riesgos, mejorando así la resiliencia urbana a través de la mejora de sus medios de vida.

Este estudio se propuso entonces re-interrogar las relaciones entre los indicadores de precariedad de asentamientos y los factores del riesgo de desastres, y avanzar así hacia nuevos planteamientos que permitan integrar los avances que se han consolidado en los estudios de riesgo de desastres en América Latina. Es necesario avanzar en este camino, construir sobre las reflexiones de este estudio, diseñar e implementar instrumentos que respondan a la complejidad de la problemática expuesta, empleando umbrales de alarma de precariedad en sus diferentes dimensiones, para informar oportunamente la toma de decisiones.

\section{REFERENCIAS}

Acolin, A. \& Green, R. (2015). Measuring housing affordability in Sao Paulo Metropolitan Region. Disponible en: https://ssrn.com/abstract=2553488 [Visitado 15 Jul 2018]

Atria, J. \& Pérez, I. (2015). Catastros de asentamientos precarios en América Latina: consideraciones metodológicas e implicancias de política. Revista de temas contemporáneos sobre lugares, política y cultura, 5(2), 208-236. Disponible en: http://www.redalyc.org/articulo.oa?id=419544926004 [Visitado 15 Jul 2018]

Candia, D. (2005). Metas del Milenio y tugurios: una metodología utilizando datos censales. Serie población y desarrollo 63. Santiago, Chile: Centro Latinoamericano y Caribeño de Demografía (CELADE) - División de Población. ISBN: 92-1-322838-4

Castro, C. P., Ibarra, I., Lukas, M., Ortiz, J. \& Sarmiento, J. P. (2015). Disaster risk construction in the progressive consolidation of informal settlements: Iquique and Puerto Montt (Chile) case studies. International Journal of Disaster Risk Reduction, 13, 109-127.

Clair, A., Reeves, A., Mckee, M. \& Stuckler, D. (2016). Housing precariousness: its measurement and impact on health before and after the Great Recession. European Journal of Public Health, 26 (1), 243-244. 
Clair, A., Reeves, A., Mckee, M. \& Stuckler, D. (2018). Constructing a housing precariousness measure for Europe. Journal of European Social Policy, o(o), 1-16.

Covarino S., Mansilla M. \& Zurita C. (2010). Guía de Diagnóstico Urbano participativo para asentamientos precarios con enfoque en la meta 11 de los objetivos del milenio. Ciudad de Guatemala, Guatemala: Universidad Rafael Landivar. ISBN: 978-9929-554-72-6

Fondation Abbé Pierre. (2018). État du mal-logement en France 2018. Rapport annuel 23. Partie I: Le mal-logement. France: Fondation Abbé Pierre. Disponible en: http://www.fondation-abbe-pierre.fr/ nos-actions/comprendre-et-interpeller/23e-rapport-sur-letat-du-mal-logement-en-france\#telechargement\%2023e\%2orapport\%202018 [Visitado 15 Jul 2018].

Ibañez, I., Flores, P., Correa, J. \& Tapia, J. M. (2015). Datos duros de una realidad muchísimo más dura. Informe encuesta Nacional de Campamentos 2015. Santiago, Chile: Centro de Investigación Social de TECHO-Chile.

Instituto Nacional de Estadísticas e informática. (2007). Perú: Mapa del Déficit Habitacional a Nivel Distrital, 2007. Lima, Perú: Dirección Técnica de Demografía y Estudios Sociales del Instituto Nacional de Estadística e Informática.

Jirón, P., Toro, A., Caquimbo, S., Goldsack, L., Martinez, L., Colonelli, P. et al. (2004). Bienestar habitacional. Guía de diseño para un hábitat residencial sustentable. Santiago, Chile: Instituto de la Vivienda, F.A.U., U. de Chile. ISBN: 956-19-0444-6

Leon, X. (2014). Barrio Mío. Transformando comunidades de alto riesgo a comunidades seguras, productivas y resilientes con la participación activa de familias y autoridades en la búsqueda de un desarrollo sostenible. Presentación no publicada, Project Concern International (PCI), Guatemala.

Mac Donald, J. (2004). Pobreza y precariedad del hábitat en ciudades de América Latina y El Caribe. Serie manuales 38. Santiago, Chile: División de Desarrollo Sostenible y Asentamientos Humanos, Comisión Económica para América Latina y el Caribe. ISBN: 92-1-322606-3

Mallett, S., Bentley, R., Baker, E., Mason, K., Keys, D., Kolar, V. et al. (2011). Precarious housing and health inequalities: What are the links?. Australia: Hanover Welfare Services, University of Melbourne, University of Adelaide, Melbourne Citymission. ISBN: O97-571773-1

Mansilla, E. (200o). Riesgo y ciudad. [Tesis Doctoral]. Universidad Autónoma de México, Ciudad de México, México. Disponible en: http://www.desenredando.org/public/libros/20oo/ryc/ RiesgoYCiudad-1.0.1.pdf [Visitado 15 Jul 2018].

Mckee, M., Clair, A., Reeves, A. \& Stuckler, D. (2017). Living on the edge: precariousness and why it matters for health. Archives of Public Health, 75, 13. DOI: 10.1186/s13690-017-0183-y

Mertins, G. (2009). Megacities in Latin America: Informality and Insecurity as Key Problems of Governance and Regulation. Die Erde, 140 (4), 391-402.

Miles, S. B., Green, R. A., \& Svekla, W. (2012). Disaster risk reduction capacity assessment for precarious settlements in Guatemala City. Disasters, 36 (3), 365-381.

Ministerio de Desarrollo Social del Gobierno de Chile. (2018). Observatorio social. Definiciones e indicadores. Vivienda. Disponible en: http://observatorio.ministeriodesarrollosocial.gob.cl/casen/ casen_def_vivienda.php [Visitado 15 Jul 2018].

Mitigation Framework Leadership Group (MitFLG). (2016). Draft Interagency Concept for Community Resilience Indicators and National-Level Measures. U.S. Department of Homeland Security. Disponible en: https://www.fema.gov/media-library/assets/documents/117607 [Visitado 15 Jul 2018].

Nichols, N. \& Braimoh, J. (2016). Community safety, housing precariousness and processes of exclusion: An institutional Ethnography from the standpoints of youth in an "unsafe" urban neighbourhood. Critical Sociology, 44(1), 157-172. DOI: 10.1177/o896920516658941

Prieto, M. (2007). Condiciones habitacionales y calidad de vida urbana. El caso de la ciudad de Bahía Blanca. IX Jornadas Argentinas de Estudios de Población. Asociación de Estudios de Población de la Argentina, Huerta Grande, Córdoba. Disponible en: https://www.aacademica.org/ooo-028/121 [Visitado 15 Jul 2018].

Sandoval, V. \& Sarmiento, J. P. (2018). Una mirada desde la gobernanza del riesgo y la resiliencia urbana en América Latina y el Caribe: Los asentamientos informales en la Nueva Agenda Urbana. Revista de Estudios Latinoamericanos sobre Reducción del Riesgo de Desastres REDER, 2(1), 38-52. 
Tapia, R. (200o). Medición de la precariedad en asentamientos urbanos irregulares. Estudio comparativo entre Venezuela, Chile y Argentina. Boletín INVI, 15 (40), 39-58.

UN-Habitat. (2015). HABITAT III Issue Papers 22 - Informal Settlements. New York: United Nations

Wellesley Institute (2010). Precarious housing in Canada. Toronto, Canada: Wellesley Institute. Disponible en: http://www.wellesleyinstitute.com/wp-content/uploads/2010/o8/Precarious_ Housing_In_Canada.pdf [Visitado 15 Jul 2018]. 